\title{
Electronic Transport in Graphene with Aggregated Hydrogen Adatoms
}

\author{
Fernando Gargiulo, ${ }_{1}^{1}$ Gabriel Autès, ${ }^{1}$ Naunidh Virk, ${ }^{1}$ Stefan Barthel,,${ }^{2,3}$ Malte Rösner, ${ }^{2,3}$ \\ Lisa R. M. Toller, ${ }^{1}$ Tim O. Wehling, ${ }^{2,3}$ and Oleg V. Yazyev ${ }^{1}$ \\ ${ }^{1}$ Institute of Theoretical Physics, Ecole Polytechnique Fédérale de Lausanne (EPFL), CH-1015 Lausanne, Switzerland \\ ${ }^{2}$ Institut für Theoretische Physik, Universität Bremen, Otto-Hahn-Allee 1, D-28359 Bremen, Germany \\ ${ }^{3}$ Bremen Center for Computational Materials Science, Am Fallturm 1a, D-28359 Bremen, Germany
}

(Received 25 July 2014; published 9 December 2014)

\begin{abstract}
Hydrogen adatoms and other species covalently bound to graphene act as resonant scattering centers affecting the electronic transport properties and inducing Anderson localization. We show that attractive interactions between adatoms on graphene and their diffusion mobility strongly modify the spatial distribution, thus fully eliminating isolated adatoms and increasing the population of larger size adatom aggregates. Such spatial correlation is found to strongly influence the electronic transport properties of disordered graphene. Our scaling analysis shows that such aggregation of adatoms increases conductance by up to several orders of magnitude and results in significant extension of the Anderson localization length in the strong localization regime. We introduce a simple definition of the effective adatom concentration $x^{\star}$, which describes the transport properties of both random and correlated distributions of hydrogen adatoms on graphene across a broad range of concentrations.
\end{abstract}

DOI: 10.1103/PhysRevLett.113.246601

PACS numbers: 72.80.Vp, 71.23.An, 73.20.Hb

Graphene has unveiled a plethora of unconventional transport phenomena [1-3], such as the universal minimal conductivity [4], Klein tunneling [5], and the anomalous quantum Hall effect $[6,7]$. On the applied side, graphene is interesting because of its exceptionally high charge-carrier mobility, which is typically limited by the presence of various types of disorder. Resonant scattering impurities, such as chemical functionalization defects [8] and dislocations [9], show the most pronounced effects on chargecarrier transport in graphene. Hydrogen adatoms represent a prototypical resonant scattering impurity, which can be experimentally introduced in a controlled fashion [10] and allows for a simple theoretical description [11]. A hydrogen adatom covalently binds to a single carbon atom of graphene resulting in rehybridization into the $s p^{3}$ state, thus effectively removing that site from the honeycomb network of $p_{z}$ orbitals. This gives rise to a zero-energy state localized around the defect, and results in the resonant scattering of charge carriers.

At a fundamental level, the classical scaling theory of Anderson transition predicts complete localization of the electronic spectrum in two dimensions, regardless of the amount of disorder [12]. For hydrogenated graphene, a model based on massless Dirac fermions with $\delta$-function point potentials confirms this prediction of the unitary class, though in 2D systems localization lengths can be strongly energy-dependent and, eventually, very large [13]. However, no unanimous consensus has been reached since experiments on hydrogenated graphene point towards metal-insulator transition, theoretically justified by the presence of electron-hole puddles (2D percolation class) [14-17].
Early works treating finite concentrations of resonant impurities in graphene assumed that the total scattering cross section deviates little from the incoherent addition of the individual cross sections, for example in the Boltzmann equation framework [18]. This picture is valid for low defect concentrations, low charge-carrier densities, and random adatom distributions. A better description requires including the effect of coherent superposition of wave functions scattered by distinct adatoms [8,19-21]. This is particularly important when impurities are in proximity to each other, with the limiting case being the formation of compact clusters in which hydrogen adatoms populate neighboring carbon atoms [22-24]. Indeed, the overall shortrange attractive interaction between individual hydrogen adatoms on graphene [25,26], combined with their relatively high diffusion mobility at room temperature [27-29], suggests a high degree of spatial correlation between adatoms.

In this Letter, we address the effects of spatial correlation of resonant impurities on electronic transport in graphene. The equilibrium configurations of hydrogen adatoms on graphene, obtained by means of Monte Carlo simulations, show a strong tendency towards aggregation into small clusters essentially eliminating isolated adatoms. Electronic transport properties investigated using the LandauerBüttiker approach complemented with kernel polynomial method calculations show that aggregation dramatically increases both the conductivity and the localization length. We propose a unified framework to account for the effects of spatial correlation of resonant scattering centers on electronic transport in graphene.

Upon adsorption, a hydrogen adatom covalently binds to a single carbon atom of graphene changing its 
hybridization state to $s p^{3}$ and its coordination sphere to tetrahedral as shown in Fig. 1(a) [30]. The covalent binding of a second adatom to the nearest neighbor carbon atom partially releases the elastic energy due to the change of coordination sphere, thus resulting in effective attractive interaction between adatoms $[25,26]$. This suggests that the interaction between adatoms can be accurately described using a short-ranged pair potential. In our study, we expand an Ising-like interaction energy $E$ up to the second nearestneighbor term

$$
E=\gamma_{1} \sum_{\langle i, j\rangle} s_{i} s_{j}+\gamma_{2} \sum_{\langle\langle i, j\rangle\rangle} s_{i} s_{j}
$$

where $\gamma_{1}$ and $\gamma_{2}$ are the corresponding first and second nearest-neighbor parameters. Here, $s_{i}=1$ if a carbon atom $i$ is populated by an adatom, otherwise $s_{i}=0$. Parameters $\gamma_{1}$ and $\gamma_{2}$ are obtained by fitting the interaction energies of adatoms calculated from first principles [31] for a set of small adatom aggregates shown in Fig. 1(b). Under the assumption of single-side functionalized graphene, the obtained parameters $\gamma_{1}=-1.182 \mathrm{eV}$ and $\gamma_{2}=0.484 \mathrm{eV}$ [41] signify a considerable first-nearest-neighbor attraction alongside a weaker second-nearest-neighbor repulsion. The excellent agreement between the interaction energy $\tilde{E}$, estimated using the fitted $\gamma_{1}$ and $\gamma_{2}$, and the first-principles values $E_{\mathrm{DFT}}$, confirms the applicability of the short-range pair potential form (1) for describing small clusters [Fig. 1(c)].

In order to assess the effect of interaction between adatoms on their spatial distribution and transport properties we perform Monte Carlo simulations using the introduced pair potential (1). The simulations employ a Monte Carlo move based on the displacement of a randomly selected adatom to a random unoccupied carbon atom in combination with the Metropolis acceptance criterion [42]. We considered models containing up to

(a)

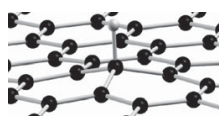

(b)

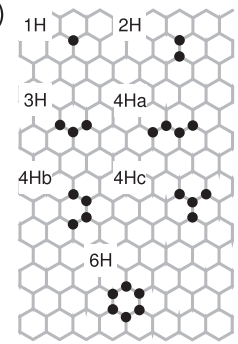

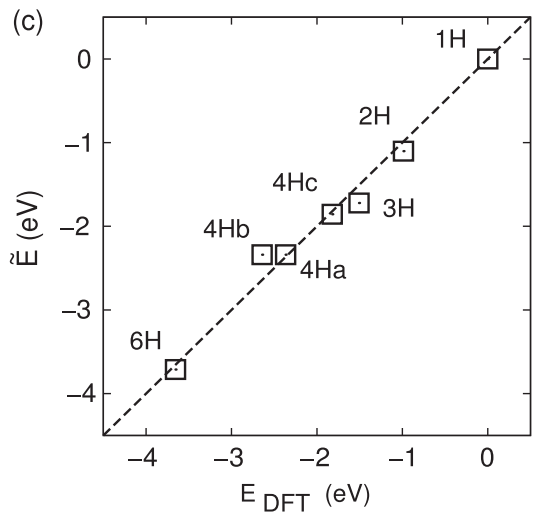

FIG. 1. (a) Atomic structure of a hydrogen adatom covalently bound to graphene. (b) Structures of small clusters of hydrogen adatoms used for fitting the pair potential of Eq. (1). (c) Predicted energy $\tilde{E}$ of aggregation of hydrogen adatoms as a function of aggregation energy $E_{\mathrm{DFT}}$ calculated from first principles for the set of adatom clusters shown in (b).
$N_{\mathrm{C}}=10^{6}$ carbon atoms $\left(165 \times 165 \mathrm{~nm}^{2}\right)$ and adatom concentrations $x=N_{\mathrm{H}} / N_{\mathrm{C}}$ ranging from $0.1 \%$ to $10 \%$. All simulations have been performed at $T=300 \mathrm{~K}$. A representative configuration of randomly distributed adatoms [Fig. 2(a)] is compared with a configuration obtained from a Monte Carlo simulation [Fig. 2(b)] at $x=5 \%$. Further details are revealed by comparing the cluster size distributions $P(n)$ [Fig. 2(c)], with adatoms populating neighboring carbon atoms being assigned to the same cluster. In the case of a random distribution, most adatoms are isolated $(n=1)$, while the occurrence of clusters $(n>1)$ is merely a probabilistic effect. In contrast, no isolated adatoms are found in the presence of interactions, with the most abundant species being adatom dimers $(n=2)$. The size distribution for the correlated case shows a longer tail with a significant probability of observing up to $n=6$ clusters. The dependence of $P(n)$ on adatom concentration $x$ is relatively weak [Fig. 2(d)].

We now focus on electronic and transport properties calculated using the nearest-neighbor tight-binding Hamiltonian for $p_{z}$ orbitals

$$
H=-t \sum_{\langle i, j\rangle}\left[c_{i}^{\dagger} c_{j}+\text { H.c. }\right]
$$

with the hopping integral constant $t=2.7 \mathrm{eV}$ [2]. An adsorbed hydrogen atom is modeled by excluding the $p_{z}$ orbital of the carbon atom to which it is bound as a
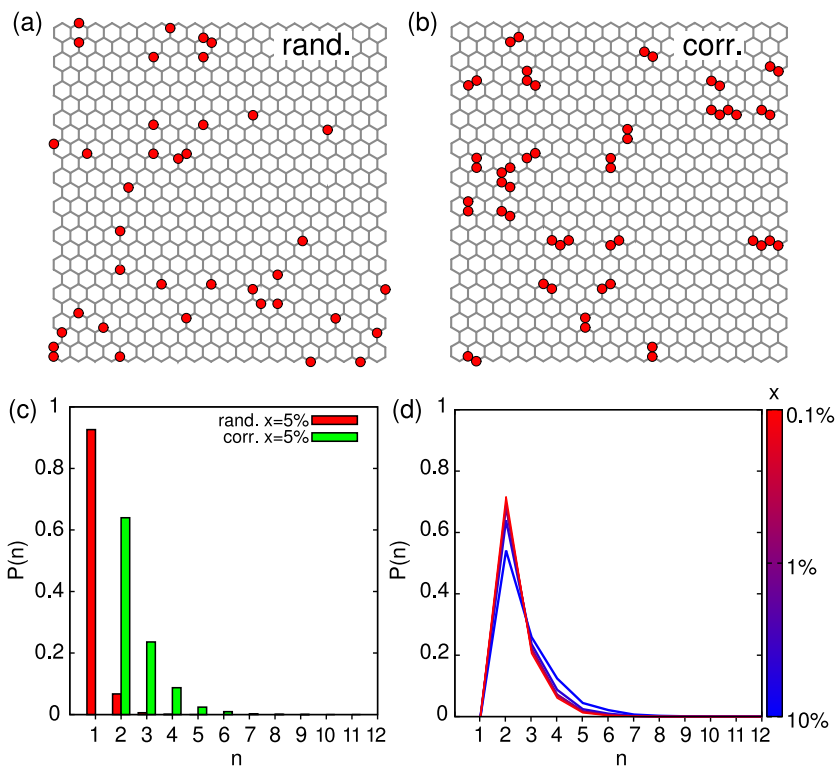

FIG. 2 (color online). Representative configurations of (a) randomly distributed and (b) correlated hydrogen adatoms on graphene at $x=5 \%$ concentration. (c) Comparison of random and correlated adatoms cluster size distributions $P(x)$ at $x=5 \%$ concentration. (d) Cluster size distributions $P(x)$ of correlated hydrogen adatoms at different concentrations. All correlated configurations are obtained by means of Monte Carlo simulations carried out at $T=300 \mathrm{~K}$. 
consequence of $s p^{3}$ hybridization, making it similar to a vacancy defect $[27,30,43]$. We stress that adatoms do not introduce coupling between the sites owing to the same sublattice of the bipartite lattice of graphene, thus maintaining electron-hole symmetry of the electronic spectrum. Figures 3(a) and 3(b) compare the density of states (DOS) of graphene with random and correlated distributions of adatoms at different concentrations. In the case of a random distribution, one observes a strong peak at $E=0$ due to the resonant modes originating from isolated adatoms $[19,43]$. The corresponding wave function is localized on the sublattice opposite to that of the carbon atom binding the adatom and decays from the defect position [44]. At high adatom concentrations, $x>1 \%$, the $E=0$ peak is accompanied by flat density regions at higher energies with a noticeable overall renormalization of the DOS, in agreement with previous calculations [43]. In comparison, the DOS calculated for the correlated impurity configurations shows a less intense peak at $E=0$ and an increased weight for $-0.9 t<E<0.9 t$ that is more evident at higher concentrations. This change is a direct consequence of different cluster size distributions. The dominant cluster type in the case of the correlated adatom distribution is the dimer $(n=2)$, which is known to be nonresonant $[11,24,45]$, meaning that no localized states emerge at any energy. The local density of states (LDOS) calculated on neighboring atoms of an isolated single adatom shows a singularity at $E=0$ [Fig. 3(c)]. In contrast, an enhancement of the LDOS in a broad energy region $-t<E<t$ is
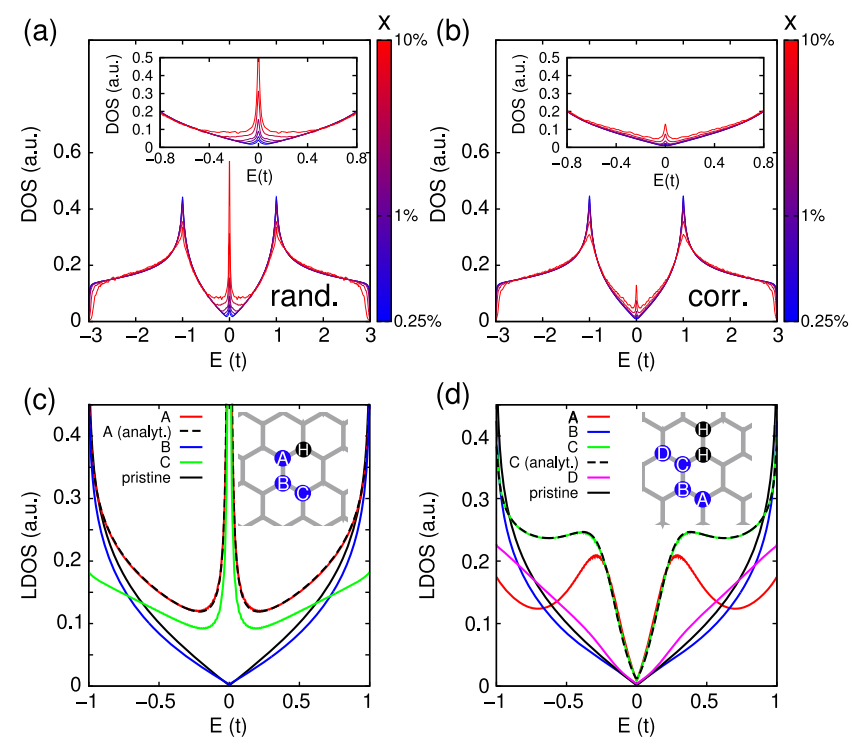

FIG. 3 (color online). Density of states of graphene in the presence of (a) randomly distributed and (b) correlated hydrogen adatoms at different concentrations. Local density of states on carbon atoms in the vicinity of (c) an isolated hydrogen adatom and (d) a dimer of hydrogen adatoms. In panels (c) and (d) LDOS referred to as "analyt." have been obtained using the analytical Green's function calculations [45]. observed on certain carbon atoms in the vicinity of the adatom dimer [Fig. 3(d)]. The numerical LDOS for selected impurities compared to the results of analytical Green's function calculations shows no discrepancies [45].

Based on these observations we conclude that the residual peak at $E=0$ in the case of the correlated distribution is due to the $n>2$ adatom aggregates, which locally break the bipartite symmetry of graphene (that is, populate different number of sites in the two sublattices). This gives rise to resonant modes at $E=0$ akin to isolated adatoms. All odd- $n$ aggregates and certain configurations of even- $n$ clusters lead to resonant modes at $E=0$. Judging by the cluster size distributions [Fig. 2(d)], the largest contribution comes from adatom trimers. This suggests that adatom aggregation has strong effects on the electronic transport properties, which are governed by resonant scattering resulting from the local bipartite symmetry breaking by adatoms and their aggregates. We investigate the electronic transport properties by performing a scaling analysis of conductivity $g$ using the Landauer-Büttiker approach [46]. In this approach, the conductance $G(E)$ is given as $G(E)=G_{0} T(E)$, where $G_{0}$ is the conductance quantum and $T(E)$ is the transmission probability across the scattering region at energy $E$. We assume a two-terminal device configuration with a scattering region of width $W=40 \mathrm{~nm}$ perpendicular to the current direction, and of variable length $1 \mathrm{~nm}<L<60 \mathrm{~nm}$. The scattering region is attached to pristine graphene contacts and populated by adatoms according to concentrations and statistical distributions discussed above. Further details of our methodology are given in the Supplemental Material [31].

The characteristic functional laws for the conductivity $g=$ $G \times L / W$ in the ballistic, diffusive, and localized transport regimes are $g \propto L, g=$ const and $g \propto \exp \left(-L / \xi_{\text {loc }}\right)$, respectively, where $\xi_{\text {loc }}$ is the localization length. In the localized regime $\ln (g)$ follows a broad positively skewed distribution, which means that $g$ can show strong fluctuations depending on the exact configuration of defects, especially in the presence of strong localization [47]. An estimate of the mean value for such a distribution is given by the typical conductivity $g_{\text {typ }}=\exp \langle\ln (g)\rangle[47,48]$. In our scaling analysis, $g_{\text {typ }}$ has been obtained averaging over 9600 disorder realizations. Figures 4(a) and 4(b) show $g_{\text {typ }}$ as a function of scattering region length $L$ at different energies for the random and correlated impurity distributions, both at $x=5 \%$ concentration. We observe a short transition from ballistic to diffusive and subsequently to localized regime within the first $10 \mathrm{~nm}$. The crossover lengths are expected to be of the order of the elastic mean free path $\xi_{\text {el }}$ and localization length $\xi_{\text {loc }}$, respectively. The general trend is that the localized regime is accentuated at low energy, whereas at higher energy the onset of exponential decay occurs at larger $L$ and the absolute slope of the conductance curves is smaller. The scaling of $g$ also depends strongly on the impurity concentration. For low adatom concentrations $(x \lesssim 0.5 \%)$ the 
onset of the localized regime is only observable in the vicinity of the Dirac energy (see Supplemental Material [31] for complete results).

We stress that the conductance curves vary smoothly in the whole range of $E$ and $x$, never showing singularities which would indicate a phase transition such as the metalinsulator transition (MIT). Thus, we ascribe the nonobservance of the localized regime to an insufficient scattering region length of our model, which is shorter than $\xi_{\text {loc }}$ for some choices of $E$ and $x$, as also pointed out in Ref. [49]. From Figs. 4(a) and 4(b) it follows that the presence of spatial correlation between adatoms enhances the conductance by up to 5 orders of magnitude in the vicinity of the Dirac point $(E=2.7 \mathrm{meV})$. This is a direct consequence of the suppressed weight of low-energy resonant states, as explained above. A closely related effect is a significant increase of the localization length $\xi_{\text {loc }}$ at all energies upon adatom aggregation. $\xi_{\text {loc }}$ was obtained by fitting conductance curves to the expected law $g_{\text {typ }} \propto \exp \left(-L / \xi_{\text {loc }}\right)$ [50], and is shown in Fig. 5(a). At $x=5 \%$, the localization length is well defined for the entire range of investigated energies $-1 \mathrm{eV}<E<1 \mathrm{eV}$, whereas at lower concentrations it is well defined only in proximity of the Dirac point (see Supplemental Material [31]). However, as long as a finite positive $\xi_{\text {loc }}$ can be determined, it proves to be up to an order of magnitude larger for the correlated adatom distribution compared to the random case.

Our Landauer-Büttiker results are complemented by the Kubo formula calculations based on the kernel polynomial
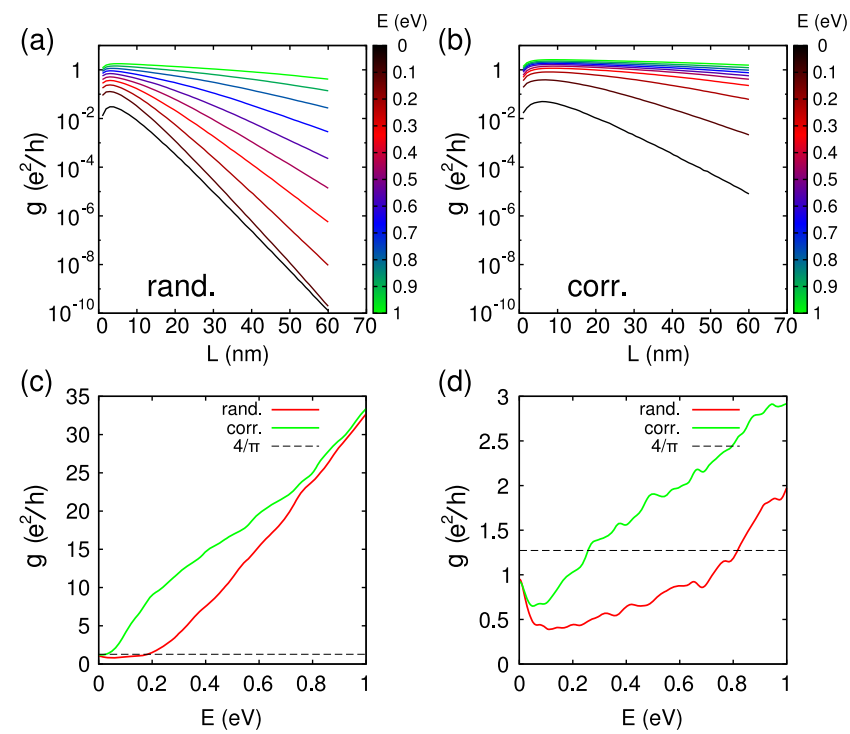

FIG. 4 (color online). (a),(b) Averaged conductivity $g_{\text {typ }}$ for the random and correlated adatoms distributions, respectively, as a function of scattering region length $L$ at different energies $E$ and at $x=5 \%$ concentration. (c),(d) Conductivity $g$ as a function of energy $E$ calculated using the kernel polynomial method for both adatom distributions at concentration $x=0.5 \%$ and $x=5.0 \%$, respectively. The dashed lines show the minimum conductivity $g=4 / \pi$. method (KPM) [51]. This approach allows for a direct calculation of the DOS and conductivity independent of sample geometry and contacts. Figures 4(c) and 4(d) show the conductivity $g$ for adatom concentrations $x=0.5 \%$ and $x=5 \%$, and both random and correlated adatom distributions. In comparison to the Landauer-Büttiker ansatz, the results are similar at both concentrations, confirming the enhancement of conductivity upon aggregation of adatoms. In addition, one can observe a monotonic increase of the conductivity and, consequently, localization length with energy. Following the classical Boltzmann theory, this is due to the inverse proportionality of conductivity to the $T$ matrix [8]. Equivalently, this refers to reduced scattering as the Fermi energy moves away from an impurity resonance. We note, however, that the type of disorder affects the energy dependence of the localization length as reported in Refs. [49] and [52]. The only qualitative difference between the Landauer-Büttiker and KPM results appears when the charge-carrier energy $E$ falls into the resonant peak region where the KPM conductivity exceeds the LandauerBüttiker results. This can be proven to be an effect of the increased DOS due to the formation of an impurity band. While the KPM conductivity reflects the excess of states at the Fermi energy, in the Landauer-Büttiker ansatz the number of charge carriers is limited by the DOS of the pristine graphene leads, and subsequently the conductivity is determined only by the mean free path and the localization length of the scattering region. We note that upon doping the leads, the LB conductivity at low energies increases while the localization length is unaffected (see Supplemental Material [31]).

On the basis of the identification of resonant adatom clusters we introduce an effective concentration

$$
x^{\star}=\frac{1}{N_{\mathrm{C}}} \sum_{i} N_{i}\left|n_{i}^{\mathrm{A}}-n_{i}^{\mathrm{B}}\right|
$$
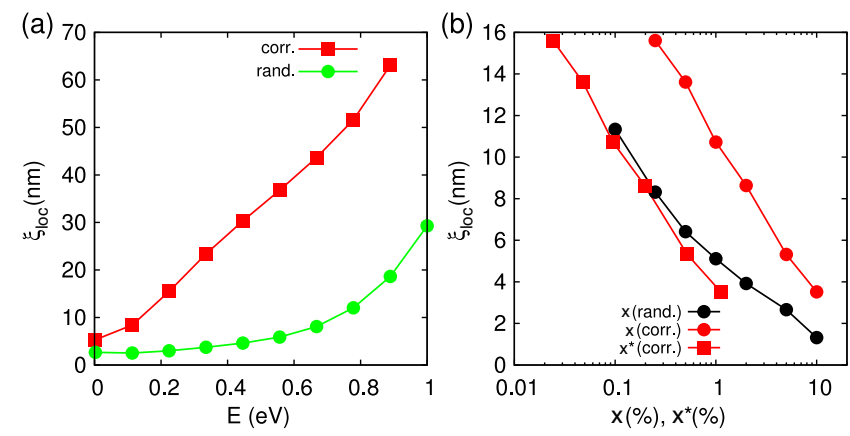

FIG. 5 (color online). (a) Localization length $\xi_{\text {loc }}$ as a function of charge-carrier energy $E$ for the case of random and correlated adatom distribution at $x=5 \%$. (b) Charge-carrier localization length $\xi_{\text {loc }}$ at low energy $\left(E=10^{-3} t=2.7 \mathrm{meV}\right)$ as a function of simple concentration $x$ and effective concentration $x^{\star}$ for the random and correlated distributions. 
where $N_{i}$ is the number of instances of the cluster configuration $i$, and $n_{i}^{\mathrm{A}}\left(n_{i}^{\mathrm{B}}\right)$ is the number of adatoms bound to carbon atoms in sublattice $A(B)$ in this configuration. As discussed above, the main contribution to $x^{\star}$ comes primarily from adatom trimers, the smallest clusters locally breaking the bipartite symmetry of graphene. We assume that the contribution of nonresonant clusters to the total scattering cross section can be neglected. It should be stressed that this approach accounts for the diverse scattering effect of different clusters. In order to validate the applicability of effective concentration $x^{\star}$, we compare $\xi_{\text {loc }}(x)$ with $\xi_{\text {loc }}\left(x^{\star}\right)$ at low charge-carrier energy for the correlated impurity case [Fig. 5(b)]. One can see that replacing $x$ with effective concentration $x^{\star}$ brings $\xi_{\text {loc }}\left(x^{\star}\right)$ evaluated for the case of the correlated impurity distribution in good agreement with $\xi_{\text {loc }}(x)$ calculated for the random distribution. The agreement is particularly good in the low concentration regime where randomly distributed impurities consist almost exclusively of isolated monomers. Deviations at higher adatom concentrations can be ascribed to the intercluster interference effects, which become important at the reduced average distance between the clusters.

To summarize, spatial correlation between resonant scattering impurities, such as hydrogen adatoms, has pronounced effects on the electronic transport properties of disordered graphene. Hydrogen adsorbed on graphene has a strong tendency toward aggregation, resulting in the formation of small clusters and fully eliminating isolated adatoms. Some of the larger clusters, notably trimers, are responsible for the residual resonant scattering, but the overall conductance and localization length dramatically increases upon aggregation. Within the range of parameters investigated in our work, we find no metal-insulator transition, with the graphene spectrum being fully localized. The predicted effects of adatom aggregation can be investigated experimentally by varying the temperature regimes, since the diffusion of hydrogen adatoms occurring at normal conditions can be effectively suppressed at low temperatures. Alternatively, time-resolved transport measurements should evince a rise in conductivity upon formation of adatom clusters.

We would like to thank C. W. J. Beenakker, P. Lugan and V. Savona for discussions. This work was supported by the Swiss National Science Foundation (Grant No. PP00P2_133552). T. O. W. acknowledges support from DFG SPP 1459 as well as the European Graphene Flagship.

[1] A. K. Geim and K. S. Novoselov, Nat. Mater. 6, 183 (2007).

[2] A. H. Castro Neto, F. Guinea, N. M. R. Peres, K. S. Novoselov, and A. K. Geim, Rev. Mod. Phys. 81, 109 (2009).

[3] S. Das Sarma, S. Adam, E. H. Hwang, and E. Rossi, Rev. Mod. Phys. 83, 407 (2011).
[4] J. Tworzydło, B. Trauzettel, M. Titov, A. Rycerz, and C. W. J. Beenakker, Phys. Rev. Lett. 96, 246802 (2006).

[5] M. I. Katsnelson, K. S. Novoselov, and A. K. Geim, Nat. Phys. 2, 620 (2006).

[6] Y. Zhang, Y.-W. Tan, H. L. Stormer, and P. Kim, Nature (London) 438, 201 (2005).

[7] K. S. Novoselov, A. K. Geim, S. V. Morozov, D. Jiang, M. I. Katsnelson, I. V. Grigorieva, S. V. Dubonos, and A. A. Firsov, Nature (London) 438, 197 (2005).

[8] T. O. Wehling, S. Yuan, A. I. Lichtenstein, A. K. Geim, and M. I. Katsnelson, Phys. Rev. Lett. 105, 056802 (2010).

[9] F. Gargiulo and O. V. Yazyev, Nano Lett. 14, 250 (2014).

[10] Z. H. Ni, L. A. Ponomarenko, R. R. Nair, R. Yang, S. Anissimova, I. V. Grigorieva, F. Schedin, P. Blake, Z. X. Shen, E. H. Hill, K. S. Novoselov, and A. K. Geim, Nano Lett. 10, 3868 (2010).

[11] T. O. Wehling, A. V. Balatsky, M. I. Katsnelson, A. I. Lichtenstein, K. Scharnberg, and R. Wiesendanger, Phys. Rev. B 75, 125425 (2007).

[12] B. Kramer and A. MacKinnon, Rep. Prog. Phys. 56, 1469 (1993).

[13] F. Evers and A. D. Mirlin, Rev. Mod. Phys. 80, 1355 (2008).

[14] A. Bostwick, J. L. McChesney, K. V. Emtsev, T. Seyller, K. Horn, S. D. Kevan, and E. Rotenberg, Phys. Rev. Lett. 103, 056404 (2009).

[15] Y. Song, H. Song, and S. Feng, J. Phys. Condens. Matter 23, 205501 (2011).

[16] R. Jayasingha, A. Sherehiy, S.-Y. Wu, and G. U. Sumanasekera, Nano Lett. 13, 5098 (2013).

[17] S. Adam, S. Cho, M. S. Fuhrer, and S. Das Sarma, Phys. Rev. Lett. 101, 046404 (2008).

[18] N. Shon and T. Ando, J. Phys. Soc. Jpn. 67, 2421 (1998).

[19] T. O. Wehling, M. I. Katsnelson, and A. I. Lichtenstein, Phys. Rev. B 80, 085428 (2009).

[20] A. Ferreira, J. Viana-Gomes, J. Nilsson, E. R. Mucciolo, N. M. R. Peres, and A. H. Castro Neto, Phys. Rev. B 83, 165402 (2011).

[21] A. Cresti, F. Ortmann, T. Louvet, D. Van Tuan, and S. Roche, Phys. Rev. Lett. 110, 196601 (2013).

[22] J. J. Palacios, J. Fernández-Rossier, and L. Brey, Phys. Rev. B 77, 195428 (2008).

[23] N. Leconte, D. Soriano, S. Roche, P. Ordejon, J.-C. Charlier, and J. J. Palacios, ACS Nano 5, 3987 (2011).

[24] G. Trambly de Laissardière and D. Mayou, Phys. Rev. Lett. 111, 146601 (2013).

[25] D. W. Boukhvalov, M. I. Katsnelson, and A. I. Lichtenstein, Phys. Rev. B 77, 035427 (2008).

[26] Y. Lin, F. Ding, and B. I. Yakobson, Phys. Rev. B 78, 041402 (2008)

[27] O. V. Yazyev, Phys. Rev. Lett. 101, 037203 (2008).

[28] C. P. Herrero and R. Ramírez, Phys. Rev. B 79, 115429 (2009).

[29] M. Moaied, J. A. Moreno, M. J. Caturla, and J. J. Palacios, arXiv:1405.3165.

[30] O. V. Yazyev and L. Helm, Phys. Rev. B 75, 125408 (2007).

[31] See Supplemental Material at http://link.aps.org/ supplemental/10.1103/PhysRevLett.113.246601 for detailed description of the methodology and additional calculations, which includes Refs. [32-40]. 
[32] J. P. Perdew, K. Burke, and M. Ernzerhof, Phys. Rev. Lett. 77, 3865 (1996).

[33] D. Vanderbilt, Phys. Rev. B 41, 7892 (1990).

[34] H. J. Monkhorst and J. D. Pack, Phys. Rev. B 13, 5188 (1976).

[35] P. Giannozzi et al. J. Phys. Condens. Matter 21, 395502 (2009).

[36] J. Mathon and A. Umerski, Phys. Rev. B 63, 220403 (2001).

[37] A. Umerski, Phys. Rev. B 55, 5266 (1997).

[38] S. Sanvito, C. J. Lambert, J. H. Jefferson, and A. M. Bratkovsky, Phys. Rev. B 59, 11936 (1999).

[39] J. M. Tomczak and S. Biermann, Phys. Rev. B 80, 085117 (2009).

[40] S. Roche, Phys. Rev. B 59, 2284 (1999).

[41] In the case of hydrogen adsorption on both sides of graphene the fitted parameters are $\gamma_{1}=-1.461 \mathrm{eV}$ and $\gamma_{2}=0.342 \mathrm{eV}$. The resulting cluster size distributions, however, are very close to those obtained for single-side adsorption [31].
[42] N. Metropolis, A. W. Rosenbluth, M. N. Rosenbluth, A. H. Teller, and E. Teller, J. Chem. Phys. 21, 1087 (1953).

[43] V. M. Pereira, F. Guinea, J. M. B. Lopes dos Santos, N. M. R. Peres, and A. H. Castro Neto, Phys. Rev. Lett. 96, 036801 (2006).

[44] D. M. Basko, Phys. Rev. B 78, 115432 (2008).

[45] F. Gargiulo, G. Autès, and O. V. Yazyev (to be published).

[46] M. Büttiker, Y. Imry, R. Landauer, and S. Pinhas, Phys. Rev. B 31, 6207 (1985).

[47] D.-H. Choe and K. J. Chang, Nano Lett. 12, 5175 (2012).

[48] A. Uppstu, Z. Fan, and A. Harju, Phys. Rev. B 89, 075420 (2014).

[49] G. Schubert and H. Fehske, Phys. Rev. Lett. 108, 066402 (2012).

[50] P. A. Lee and T. V. Ramakrishnan, Rev. Mod. Phys. 57, 287 (1985).

[51] A. Weiße, G. Wellein, A. Alvermann, and H. Fehske, Rev. Mod. Phys. 78, 275 (2006).

[52] S.-J. Xiong and Y. Xiong, Phys. Rev. B 76, 214204 (2007). 